\title{
Structural basis of the therapeutic anti-PD-L1 antibody atezolizumab
}

\author{
Fei Zhang ${ }^{1, *}$, Xiaoqiang $\mathbf{Q i}^{2,{ }^{*}}$, Xiaoxiao Wang ${ }^{3, *}$, Diyang $\mathbf{W e i}^{3}$, Jiawei $\mathbf{W u}^{1}$, Lingling \\ Feng $^{1}$, Haiyan $\mathrm{Cai}^{1}$, Yugang Wang ${ }^{1}$, Naiyan Zeng ${ }^{1}$, Ting $\mathrm{Xu}^{3}$, Aiwu Zhou ${ }^{1}$ and Ying $^{3}$ \\ Zheng ${ }^{1}$ \\ ${ }^{1}$ Hongqiao International Institute of Medicine, Shanghai Tongren Hospital/Faculty of Basic Medicine, Key Laboratory of Cell \\ Differentiation and Apoptosis of The Chinese Ministry of Education, Shanghai Jiao Tong University School of Medicine, \\ Shanghai, China \\ ${ }^{2}$ Faculty of Basic Medicine, Nanjing Medical University, Nanjing, China \\ ${ }^{3}$ The Therapeutic Antibody Research Center of SEU-Alphamab, Southeast University, Nanjing, China \\ *These authors have contributed equally to this work \\ Correspondence to: Aiwu Zhou, email: awz20@shsmu.edu.cn \\ Ting Xu, email: tingxu@alphamab.com \\ Ying Zheng, email: zhengyingsh@126.com
}

Keywords: atezolizumab, PD-L1, crystal structure, immunotherapy, hot-spot residue

Received: July 14, $2017 \quad$ Accepted: August 29, $2017 \quad$ Published: October 06, 2017

Copyright: Zhang et al. This is an open-access article distributed under the terms of the Creative Commons Attribution License 3.0 (CC BY 3.0), which permits unrestricted use, distribution, and reproduction in any medium, provided the original author and source are credited.

\section{ABSTRACT}

Monoclonal antibodies targeting PD-1/PD-L1 signaling pathway have achieved unprecedented success in cancer treatment over the last few years. Atezolizumab is the first PD-L1 monoclonal antibody approved by US FDA for cancer therapy; however the molecular basis of atezolizumab in blocking PD-1/PD-L1 interaction is not fully understood. Here we have solved the crystal structure of PD-L1/atezolizumab complex at 2.9 angstrom resolution. The structure shows that atezolizumab binds the front beta-sheet of PD-L1 through three CDR loops from the heavy chain and one CDR loop from the light chain. The binding involves extensive hydrogen-bonding and hydrophobic interactions. Notably there are multiple aromatic residues from the CDR loops forming Pi-Pi stacking or cation-Pi interactions within the center of the binding interface and the buried surface area is more than $2000 \AA^{2}$, which is the largest amongst all the known PD-L1/antibody structures. Mutagenesis study revealed that two hot-spot residues (E58, R113) of PD-L1 contribute significantly to the binding of atezolizumab. The structure also shows that atezolizumab binds PD-L1 with a distinct heavy and light chain orientation and it blocks PD-1/PD-L1 interaction through competing with PD-1 for the same PD-L1 surface area. Taken together, the complex structure of PD-L1/atezolizumab solved here revealed the molecular mechanism of atezolizumab in immunotherapy and provides basis for future monoclonal antibody optimization and rational design of small chemical compounds targeting PD-L1 surface.

\section{INTRODUCTION}

Cancer immunotherapies which utilize antibodies masking the inhibitory receptor have drawn considerable attention in recent years [1-4]. Several monoclonal antibodies (MAbs) targeting CTLA-4, PD-1 and PD-L1 have been approved by FDA for clinical applications in
USA. PD-L1 (CD274, B7-H1) is expressed widely on both lymphoid and nonlymphoid tissues [5] and it is the primary PD-1 ligand. It is up-regulated in solid tumors, where it can inhibit the activity of $\mathrm{PD}-1^{+}$, tumor-infiltrating $\mathrm{CD} 4^{+}$ and $\mathrm{CD}^{+} \mathrm{T}$ cells [6-8]. Blockade of PD-L1 binding is an attractive strategy for restoring tumor-specific T-cell immunity $[9,10]$. Tumor responses have been obtained 
both with anti-PD-1 and anti-PD-L1 therapies in patients with several forms of cancer [11-15].

Atezolizumab (TECENTRIQ) developed by Genetech is the first therapeutic anti-PD-L1 antibody approved in the United States for the treatment of patients with metastatic urothelial carcinoma or non-small cell lung cancer who have progressed during or following platinum-containing chemotherapy [https://www.fda. gov/NewsEvents/Newsroom/Press Announcements/ ucm501762.htm]. There are more than forty ongoing trials with atezolizumab either as monotherapy or in combination with other agents (e.g., bevacizumab, cobimetinib, obinutuzumab, bendamustine, ipilimumab, interferonalfa). Clinical indications under investigation include renal cell carcinoma, hepatocellular carcinoma, triple negative breast cancer, colorectal cancer, hematologic malignancies in addition to other tumor types as well. Several other anti-PD-L1 antibodies including avelumab, durvalumab and KN035 are also in intensive clinical trials. Crystal structures of these PD-L1 antibodies such as avelumab, durvalumab, BMS936559 and KN035 have been reported recently [16-19], however, it is unclear how atezolizumab binds and blocks PD-1/PD-L1 pathway. In the present study, we have prepared the Fab fragment of atezolizumab and solved its crystal structure in complex with PD-L1 and analyzed its binding characteristics.

\section{RESULTS AND DISCUSSION}

\section{Overall structure of PD-L1/atezolizumab complex}

Atezolizumab Fab fragment was expressed in HEK293 expression system and purified from the culture medium. The $\mathrm{IgV}$ domain of PD-L1 was expressed in E.coli as inclusion body and purified after refolding as previously described [19]. Crystals of the PD-L1/ atezolizumab complex were grown from $2 \mathrm{M}$ ammonium sulfate in $0.1 \mathrm{M}$ Tris $\mathrm{pH} 7.0$ and the structure of this complex was solved at $2.9 \AA$ resolution with a single complex assembly in the asymmetric unit (Table 1). PD-L1 assumes a beta-sandwich immunoglobulin-variable (IgV)type topology with Cys40 and Cys 114 forming a disulfide bridge. The binding site of atezolizumab on PD-L1 is mainly located on the front $\beta$-sheet which is constituted by strands A, G, F, C, and C' of the IgV domain of PDL1 (Figure 1). The structure revealed that both heavy chain (VH) and light chain (VL) of atezolizumab interact with PD-L1. All three complementarity determining region (CDR) loops of $\mathrm{VH}$ of atezolizumab are involved while only CDR3 loop of VL forms interaction with PDL1 (Figure 2). When structure of PD-L1/atezolizumab complex is superimposed with the structure of full length PD-L1 (PDB: 5JDR) or PD-1/PD-L1 complex (PDB: $4 \mathrm{ZQK}$ ), the root-mean-square deviations (RMSDs) are $0.53 \AA$ and $0.56 \AA$ respectively, indicating no significant changes in PD-L1 structure during atezolizumab binding (Supplementary Figure 1) [19, 20].

\section{Interactions between PD-L1 and atezolizumab}

Detailed structural analysis of PD-L1/atezolizumab complex by PISA software (Proteins, Interfaces, Structures and Assemblies) shows that there are 13 hydrogen bonds (Supplementary Table 1) and about 82 contacts within $3.7 \AA$ radius in PD-L1/atezolizumab interface (Supplementary Table 2). PD-L1 residues from the strand C (D49, A52, I54, Y56, E58) and strand C'(N63, Q66, V68), the C'C loop (M59, E60, D61, K62), strand F (V111, R113, M115), FG loop (G119) as well as strand G (A121, D122, Y123, R125) form an extended surface to interact with heavy chain residues from CDR1 (S30, D31), CDR2 (Y54, G55, S57, T58), CDR3 (R99, W101) loops from the heavy chain of atezolizumab (Figure 2A, 2B). Especially, residues (Y54, G55, S57, T58) of the CDR2 loop of the heavy chain (HCDR2) form 6 hydrogen bonds with residues from strand C (E58), strand C' (N63, Q66) and CC' loop (D61) (Figure 2C), and residues from HCDR1 loop (S30, D31) form 4 polar interactions with strand F (R113) and strand G (Y123, R125) (Figure 2D). Notably the side chain of Y54 from HCDR2 loop pokes into a cleft formed by strand $\mathrm{C}$ and $\mathrm{G}$ and forms a H-bond with the main-chain oxygen atom of V111 of PD-L1, and at the same time it is further stabilized by a cationPi interaction with the side chain of R125 (Figure 2D). Furthermore the side chain of M115 of strand F in PDL1 is positioned along the hydrophobic patch formed by W33 (HCDR1), W50 (HCDR2) and W101 (HCDR3) of atezolizumab, while the adjacent $\mathrm{Y} 123$ of strand G packs with W101 (HCDR3) through $\pi$ - $\pi$ interaction (Figure 2E). Residues L92 and Y93 from LCDR3 form close contact with PD-L1 residues A51, A52 and G119. Overall, these extensive H-bonding and hydrophobic interactions result a total buried surface area of more than $2000 \AA^{2}$ in PDL1/atezolizumab binding interface, which is the largest among the known PD-L1/antibodies structures. The buried surface area of atezolizumab is $1029 \AA^{2}$ with $784 \AA^{2}$ from the heavy chain and $245 \AA^{2}$ from the light chain in the binding interface, and the buried surface area of PD-L1 is $971 \AA^{2}$ (Figure 3A). Although the buried surface area is not necessarily proportional to the binding affinity between the antibody and antigen, the large binding surface of atezolizumab may provide certain advantage in the selectivity of this antibody.

\section{Structural basis of atezolizumab in PD-1/PD-L1 blockade}

To analyze how atezolizumab would block PDL1's interactions, we systemically analyzed the binding areas of PD-L1 when it is complexed with its ligand or blocking antibodies. Interestingly we found that all the 
PD-L1/atezolizumab complex

\section{Data collection:}

Beamline

Space group

\section{Cell dimensions}

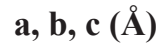

$\boldsymbol{\alpha}, \boldsymbol{\beta}, \boldsymbol{\gamma}\left({ }^{\circ}\right)$

Wavelength (Å)

Resolution (§̊)

Total NO. of observation

Total NO. unique

$\mathbf{R}_{\text {merge }}(\%)$

I/ $\mathbf{I}$

Completeness (\%)

Multiplicity

Refinement:

Resolution (Å)

No. of reflections/free

No. of residues

No. of atoms

$\mathbf{R}_{\text {work }} / \mathbf{R}_{\text {free }}$

B-factors $\left(\AA^{2}\right)$

RMSD

Bond lengths ( $(\AA)$

Bond angles $\left({ }^{\circ}\right)$

\section{Ramachandran plot}

In Preferred Region (\%)

In Allowed Region (\%)

Outliers (\%)
SSRF 17U

C121

$127.43,73.05,64.00$

$90.00,104.39,90.00$

0.9792

$2.90-61.60$ (2.90-3.08)

34659(5741)

11768 (1927)

$20.0(57.8)$

$4.6(1.8)$

$93.4(95.1)$

$2.9(3.0)$

2.90-61.60

$11744 / 545$

518

3979

$0.239 / 0.295$

45.7

0.004

0.845

94.6

5.16

0.2 binding areas on PD-L1 on located on the front beta-sheet with a buried surface area of $752.3 \AA^{2}$ for PD-1, 644.4 $\AA^{2}$ for KN035, 875.4 $\AA^{2}$ for avelumab, 742.4 $\AA^{2}$ for BMS936559 and $971 \AA^{2}$ for atezolizumab respectively. These binding areas largely overlap each other (Figure 3B). Also it appears that the heavy chain rather than the light chain in all the MAbs plays a dominant role in binding (Supplementary Figure 2). Furthermore when the structure of PD-1/PD-L1 complex (PDB: 4ZQK) is overlaid with that of PD-L1/atezolizumab complex, it is apparent that atezolizumab clashes with PD-1 and they could not bind to a same PD-L1 molecule at the same time. Therefore, atezolizumab, like other known PD-L1 antibodies, blocks PD-1/PD-L1 interaction through competing with PD-1 for the same surface area on PD-L1.

Despite these common features, there are distinct differences amongst these PD-L1 antibodies. For example, the relative positions of the heavy and light chain of the MAbs when bound on PD-L1 differ significantly. The heavy and light chains of avelumab adopt a bottom-top configuration, where the heavy chain binds the bottom half of the PD-L1 surface and the light chain binds on the top part. BMS-936559 antibody adopts a right-left configuration with atezolizumab adopting a top-bottom 
configuration. Notably the anti-PD-L1 nanobody KN035 binds PD-L1 mainly through a single CDR loop of a single $\mathrm{IgV}$ domain. Also some of these antibodies are in either IgG1 form (avelumab, durvalumab, atezolizuamb) or IgG4 isoform (BMS-936559) [21]. These differences will result differences in the orientations of these antibodies when bound on PD-L1 on the cell membrane and affect subsequent binding of Fc receptors, which will lead to differences in antitumor activities or selectivity towards different types of cancers. Obviously extensive clinical tests are required to make full use of these antibodies for the benefits of cancer patients.

It is also somewhat surprising that all these PDL1 blocking antibodies bind PD-L1 on the flat front beta-sheet as generally targeting a relatively flat protein surface is very challenging. When compared with other
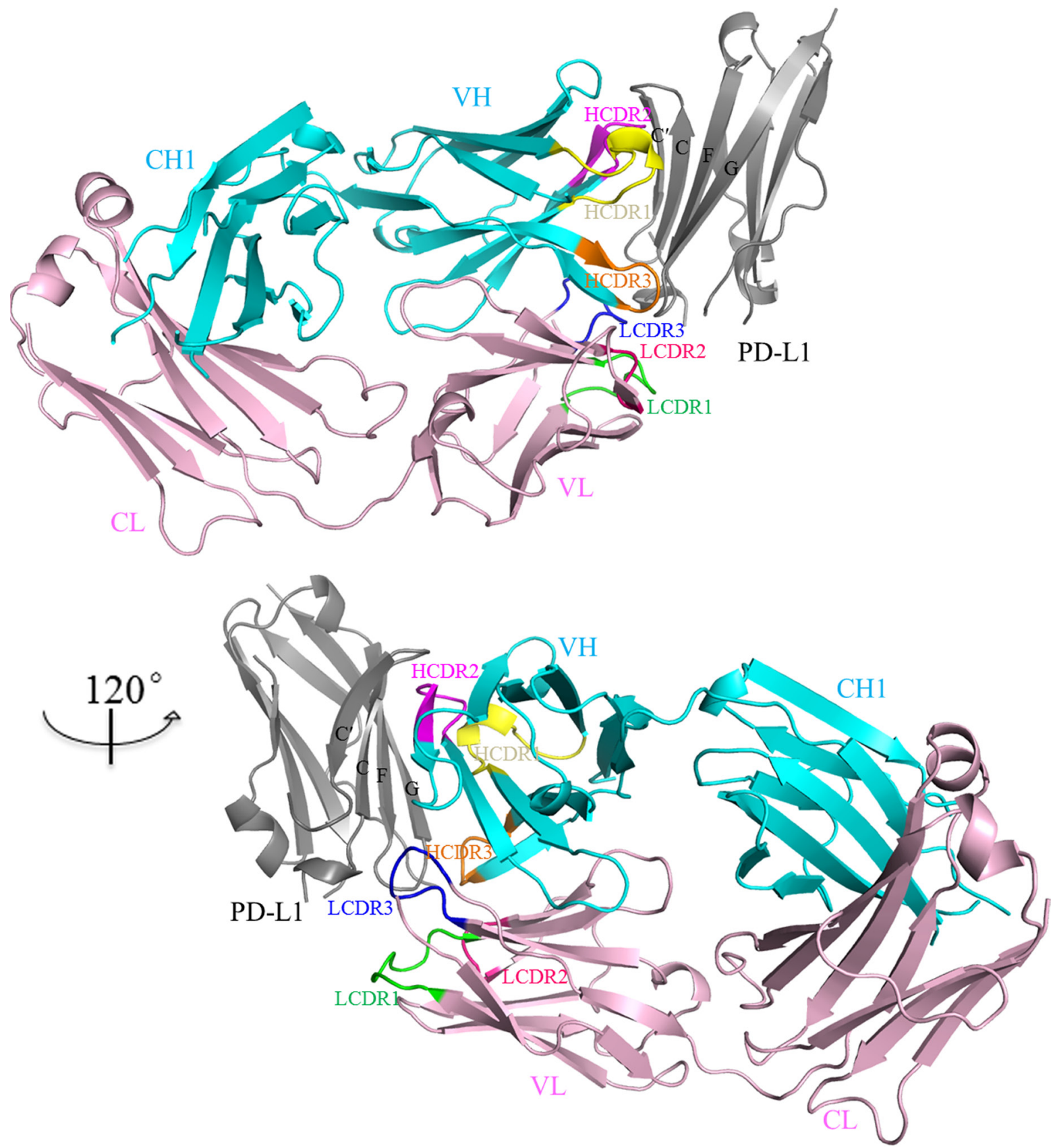

Figure 1: Overall structure of PD-L1/atezolizumab complex. The IgV domain of PD-L1 is shown in grey and the heavy chain and light chain of atezolizumab are shown in cyan and pink respectively. The CDR loops from the heavy chain are colored in yellow (HCDR1), magenta (HCDR2) and orange (HCDR3) respectively. The CDR loops from the the light chain are colored in green (LCDR1), hot pink (LCDR2) and blue (LCDR3) respectively. Atezolizumab binds the front $\beta$-sheet of PD-L1-IgV domain (grey) through three CDR loops from the heavy chain and CDR3 loop (LCDR3) from the light chain. 
A

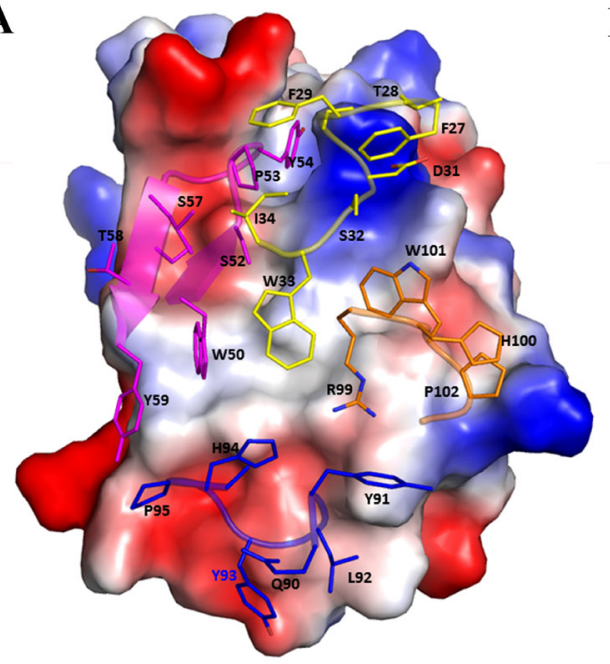

B

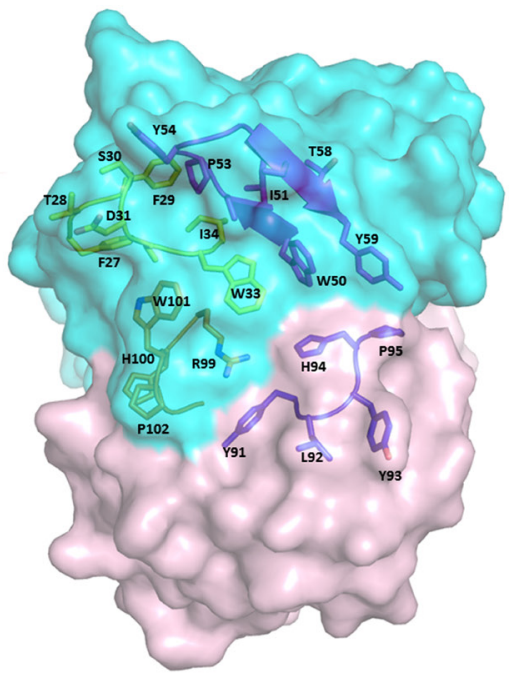

C
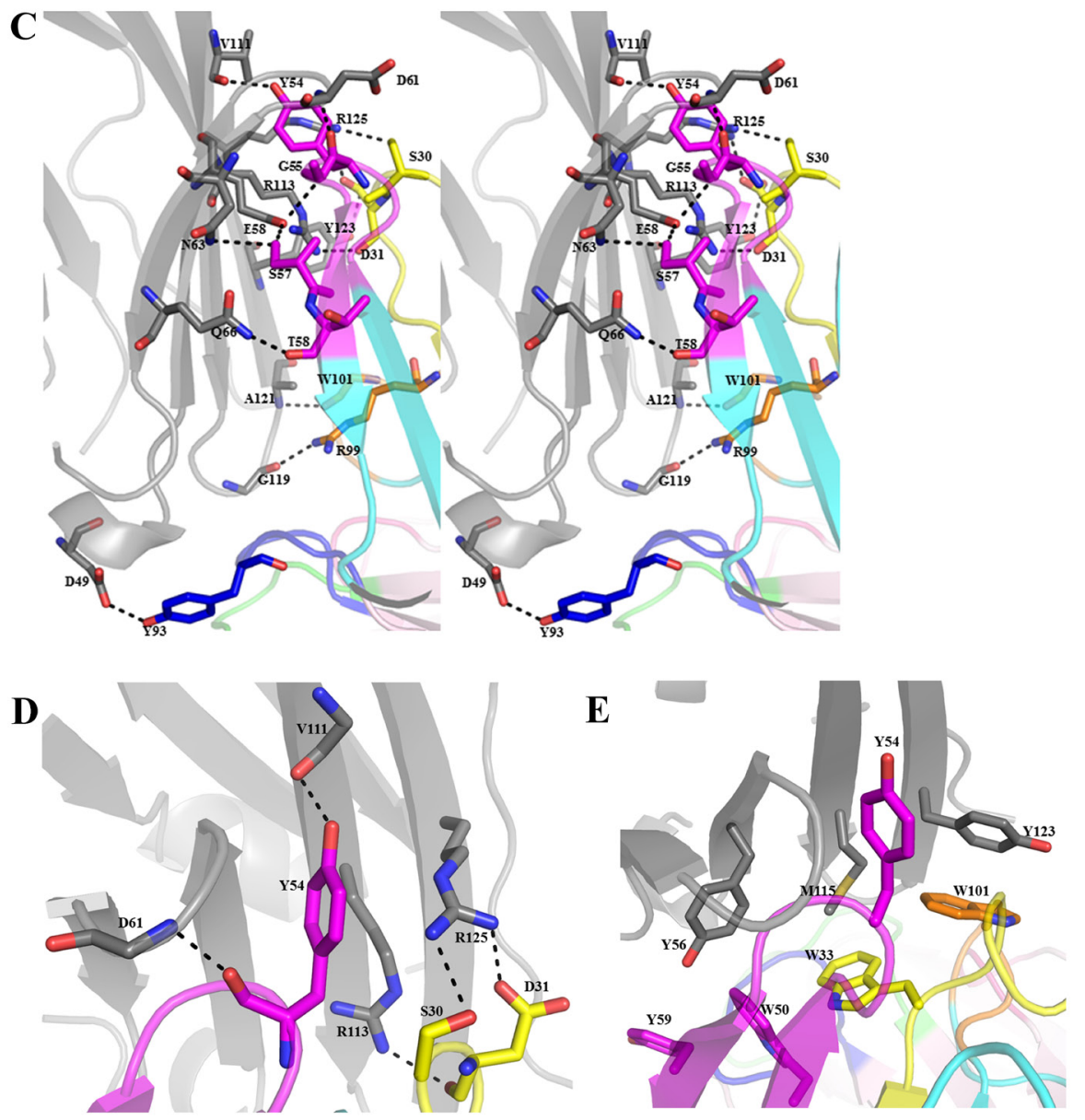

Figure 2: Detailed interactions in the interface of PD-L1/atezolimumab complex. The electrostatics surface of PD-L1 is shown in (A) with key residues from CDR loops of atezolizumab shown in sticks. The CDR loops are color with same scheme as in Figure 1 where HCDR1 is in yellow, HCDR2 in magenta, HCDR3 in orange and LCDR3 in blue. The binding surface of atezolizumab is shown in (B) with heavy chain in cyan and light chain in magenta. Some of the key residues of heavy and light chain are in sticks (B). The wall-eye stereo view of the interface of PD-L1/atezolizumab complex shows detailed hydrogen bonds between these two molecules (C). Residues involved are shown as sticks with hydrogen bonds as dashed lines.Y54 of HCDR2 is inserted into a cleft formed by CC' loop and strand G of PD-L1 forming a hydrogen bond with V111 of PD-L1 which is further stabilized by the side chain of R125 of PD-L1 through cation-Pi interaction. The side chain of R125 is in turn stabilized by S30 and D31 from HCDR1 loop (A, D). PD-L1 residues of M115 along with Y56 and Y123 form hydrophobic interactions with a hydrophobic surface formed aromatic residues W33, W50, Y59 and W101 of CDR loops of atezolizumab (E). 
A

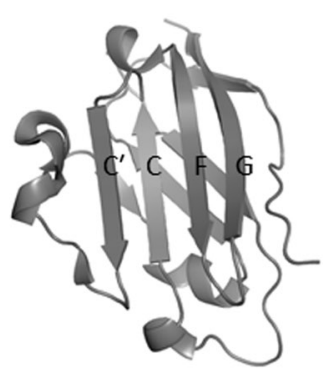

PD-L1

D

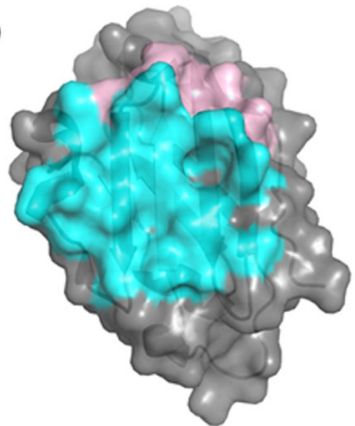

Avelumab Interface area: $875.4 \mathrm{~A}^{2}$
B

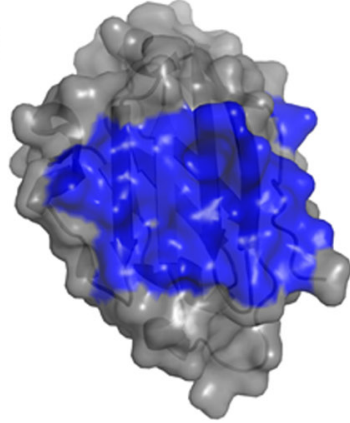

PD-1

Interface area: $752.3 \mathrm{~A}^{2}$

E

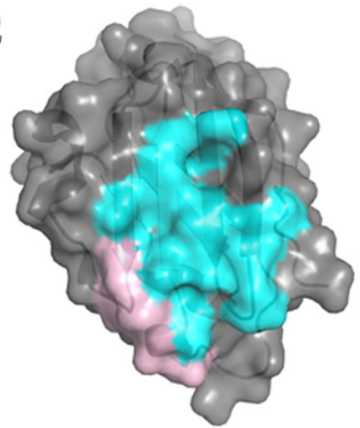

BMS-936559

Interface area: $742.4 \mathrm{~A}^{2}$

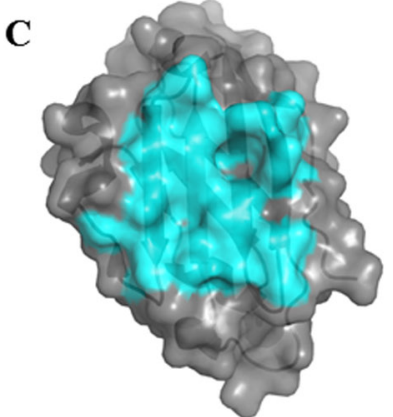

$\mathrm{KN} 035$

Interface area: $644.4 \mathrm{~A}^{2}$

F

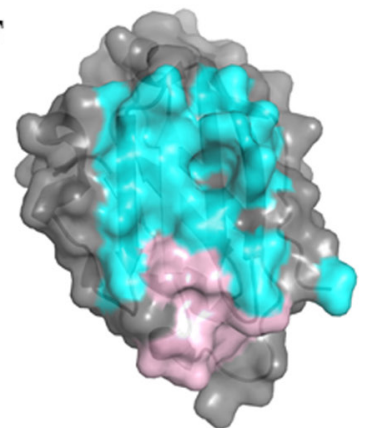

Atezolizumab Interface area: $971 \mathrm{~A}^{2}$

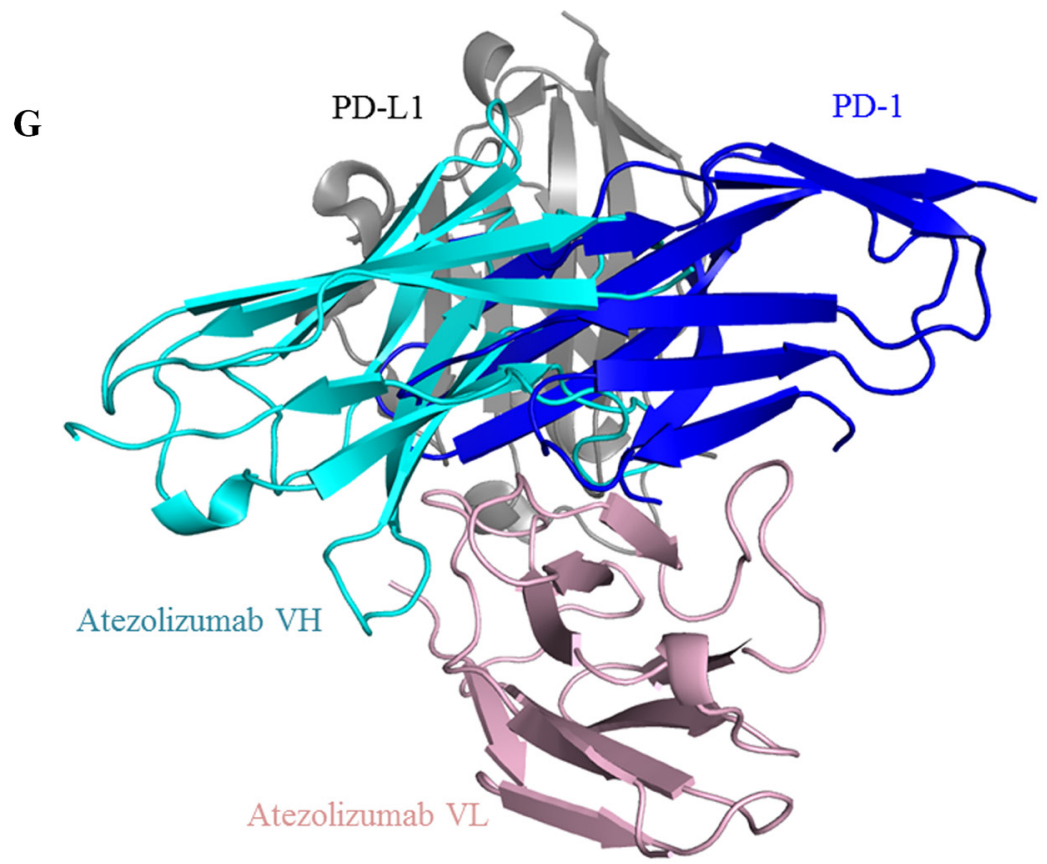

Figure 3: Comparison of the binding mode of anti-PD-L1 MAbs. The front $\beta$-sheet of PD-L1 is composed with strand C', C, F and G (A). The binding surface area that is covered by PD-1 (B), KN035 (C), Avelumab (D), BMS-936559 (E) or Atezolizumab (F) is highlighted with the covered surface area calculated. The areas covered by heavy or light chain of MAbs (D, E, F) are color in cyan and pink respectively. Superposition of the structure of PD-L1/atezolizumab complex with PD-1/PD-L1complex (PDB: 4ZQK) shows clashes between PD-1 and atezolizumab indicating that the antibody competes with PD-1 for binding to PD-L1. the CH1domain of heavy chain and CL domain of light chain were omitted (G) for clarity. 
immune checkpoint blocking antibodies, we have found that CTLA-4 blocking antibodies such as ipilimumab (PDB:5TRU) and tremelimumab (PDB:5GGV) similarly target the front surface of $\operatorname{IgV}$ domain of CTLA-4, while PD-1 blocking antibodies such as pembrolizumab (PDB:5GGS) and nivolumab (PDB:5GGR) mainly bind PD-1 on the connecting loops of the front beta-sheet [18, 22-24]. It is unclear if the differences in the epitopes of these receptors are due to the differences in antibody screening processes or due to differences in the geometry and electrostatic properties of the $\mathrm{IgV}$ domains of these receptors. Nevertheless, the distinct binding surfaces of these PD-L1 antibodies targeting same area on PDL1 revealed by these crystallography studies provide invaluable test cases for fine-tuning programs in predicting protein-protein interactions.

\section{Hot-spot residues}

It has been shown that many residues are often involved in a protein binding interface, but only a few of them make critical contributions towards complex formation. These key residues are often called the hotspot residues of a given protein surface and they are general targets for rational drug design in blocking protein interactions $[25,26]$. We have previously solved the crystal structure of an anti-PD-L1 nanobody KN035 and analyzed the contribution of each PD-L1 residue of the interface towards binding through mutagenesis and affinity measurement. The hot-spot residues of PD-L1 surface identified are I54, Y56, E58, Q66 and R113. Here we have compared the binding interfaces of all known PD-L1/antibody complexes and calculated the buried surface area of each residue in the interfaces (Figure 4). The plot shows that the binding of PD-L1 towards each antibody involves about 20-30 residues with about half of these residues significantly covered by the antibodies in the interfaces. Most significantly, the five hot-spot residues we identified are all involved in binding to these antibodies. Subsequently we tested the role of these hotspot residues in atezolizmab binding. The PD-L1 mutants, prepared in our previous study [19], were tested for their

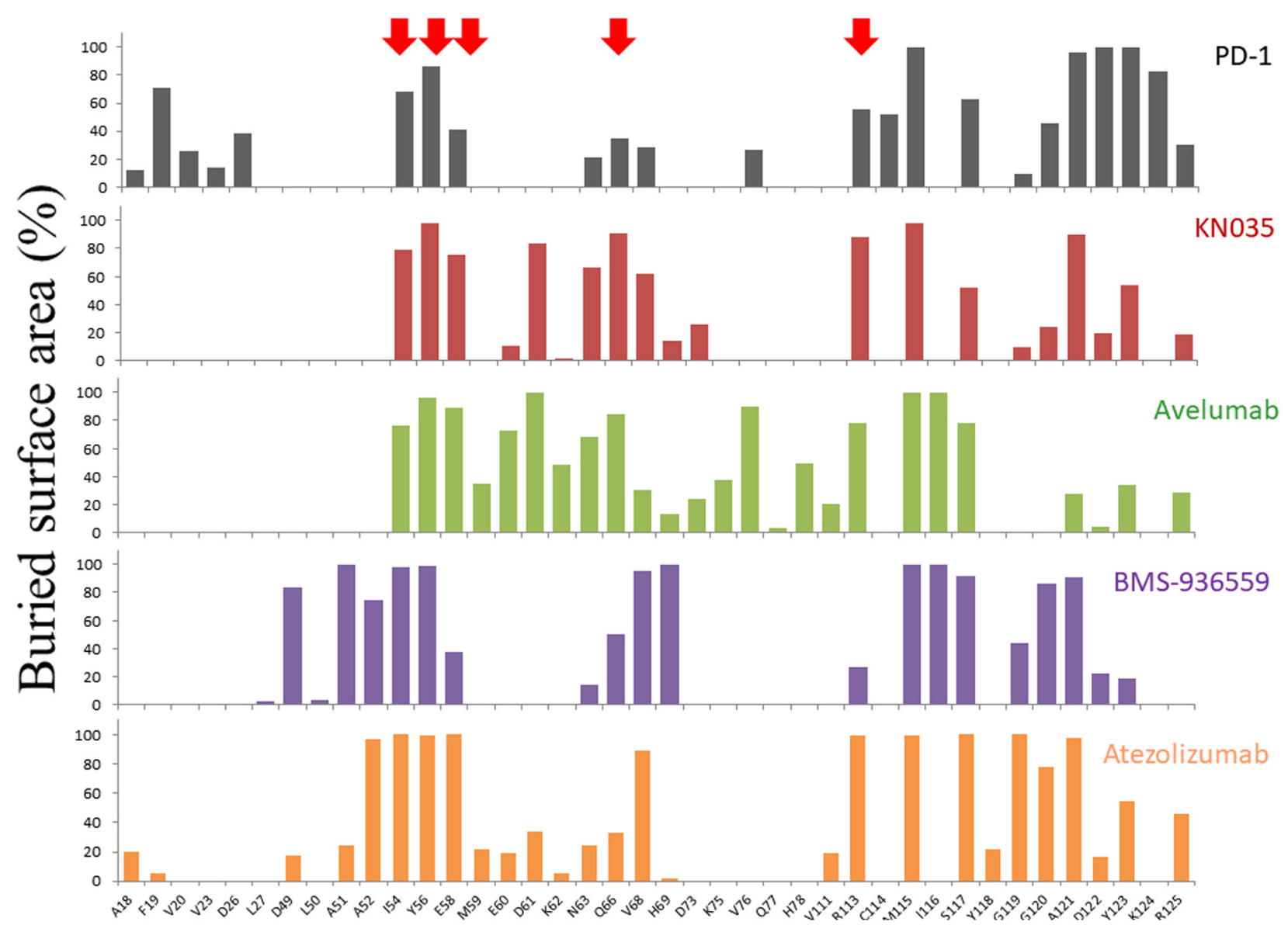

Figure 4: Buried surface areas of residues in PD-L1 binding interface. All the PD-L1 residues that are involved in binding to PD-1, KN035, Avelumab, BMS-936559 and Atezolizumab are selected with the percentage of the buried surface area of each residue plotted. The five hot-spot residues (I54, Y56, E58, Q66 and R113) identified from PD-L1/KN035 structure are labeled with red arrows. Although the structure of PD-L1/Durvalumab was also published recently, the coordinate of this structure has not been released and is not included here for comparison. 
Table 2: Binding affinities of PD-L1 variants towards KN035 and atezolizumab

\begin{tabular}{lcccc}
\hline PD-L1 variants & $\mathbf{K}_{\mathbf{d}} \mathbf{o f ~ K N 0 3 5}(\mathbf{M})$ & $\mathbf{K}_{\mathrm{d}, \text { varitant }} / \mathbf{K}_{\mathbf{d}, \mathbf{w T}}$ & $\mathbf{K}_{\mathbf{d}}$ of atezolizumab $(\mathbf{M})$ & $\mathbf{K}_{\mathbf{d}, \text { variant }} / \mathbf{K}_{\mathbf{d}, \mathbf{w T}}$ \\
\hline WT & $3.0 \mathrm{E}-09$ & 1 & $9.96 \mathrm{E}-09$ & 1 \\
I54A & $2.42 \mathrm{E}-07$ & 80.7 & $3.23 \mathrm{E}-08$ & 3.2 \\
Y56A & $1.24 \mathrm{E}-06$ & 413.3 & $2.68 \mathrm{E}-08$ & 2.7 \\
E58A & $1.49 \mathrm{E}-07$ & 49.7 & $1.81 \mathrm{E}-07$ & 18.2 \\
D61A & $1.99 \mathrm{E}-08$ & 6.6 & $9.99 \mathrm{E}-09$ & 1.0 \\
N63A & $2.30 \mathrm{E}-08$ & 7.7 & $1.73 \mathrm{E}-08$ & 1.7 \\
Q66A & $4.88 \mathrm{E}-07$ & 162.7 & $2.46 \mathrm{E}-09$ & 0.25 \\
R113A & $5.34 \mathrm{E}-07$ & 178 & $8.52 \mathrm{E}-08$ & 8.6 \\
M115A & $5.51 \mathrm{E}-08$ & 18.4 & $4.57 \mathrm{E}-08$ & 4.6 \\
Y123A & $4.24 \mathrm{E}-08$ & 14.1 & $4.66 \mathrm{E}-08$ & 4.7 \\
R125A & $2.97 \mathrm{E}-08$ & 9.9 & $5.89 \mathrm{E}-08$ & 6.0 \\
\hline
\end{tabular}

The binding affinity was determined where KN035-Fc or full-length atezolizumab was immobilized on a protein A chip and then treated with different concentrations of PD-L1 variants as previously described [19].

binding affinity towards atezolizumab (Table 2). The results showed that replacement of hot-spot residues (E58 and R113) by alanine leads to 18 and 9-fold decrease in the binding affinity of PD-L1 towards atezolizumab respectively, while other residues play a relatively minor role. This indicates that atezolizumab adopts two of the hot-spot residues from this part of PD-L1 surface for its efficient binding. Further confirmation of the importance of these PD-L1 residues comes from the recent structural study of PD-L1 with chemical PD-L1 inhibitors developed by Bristol-Myers Squibb where binding of these compounds on PD-L1 involves two hot-spot residues I54 and Y56 [27, 28]. Although it is largely unexpected that these chemical inhibitors inhibit PD-L1 binding surface through inducing dimerization of PD-L1, the hot-spot residues shown here will provide rational targets for the development of new class of chemical compounds as PDL1 inhibitors.

In conclusion, the crystal structure of therapeutic antibody atezolizumab complexed with PD-L1 solved here shows that the antibody blocks PD-1/PD-L1 pathway through competing with PD-1 for the binding surface on PD-L1 and this provides basis for further antibody optimization and for the development of novel chemical PD-L1 inhibitors for immunotherapy.

\section{MATERIALS AND METHODS}

\section{Protein purification and complex preparation}

The Fab fragment of atezolizumab was expressed in the transient HEK293 expression system. Mixture of PEI (Polyscience) and expression plasmids coding the heavy chain and light chain of the Fab fragment of atezolizumab (w/w 3:1) was transfect into HEK293 suspension cell at a density of $4^{*} 10^{6} / \mathrm{ml}$. Culture medium were collected after 3 days through centrifugation. The assembled Fab fragment of atezolizumab with a his-tag at the C-terminus of the heavy chain was purified by Ni column (GE healthcare) from the medium after dialysis against $10 \mathrm{mM}$ Tris- $\mathrm{HCl}$, $\mathrm{pH} 7.4,150 \mathrm{mM} \mathrm{NaCl}$. IgV domain of PD-L1 is prepared as previously described [19]. Briefly, the DNA sequence encoding human PD-L1 IgV domain (amino acids 19132 ) with a C-terminal His-tag was cloned into pET-28a and transformed into E.coli BL21 (DE3) as inclusion bodies. Cells were cultured at $37^{\circ} \mathrm{C}$ in LB and induced with $0.5 \mathrm{mM}$ IPTG (isopropyl- $\beta$-D-thiogalactoside) once the optical density at $600 \mathrm{~nm}$ reached 1.0. After a further 16-hour incubation at $37^{\circ} \mathrm{C}$, the cells were collected by centrifugation, resuspended in lysis buffer $(20 \mathrm{mM}$ Tris$\mathrm{HCl}, \mathrm{pH} 7.4,1 \%$ Triton $\mathrm{X}-100$, and $20 \mathrm{mM}$ EDTA) and lyzed by sonication. Inclusion bodies were recovered by centrifugation at $15,000 \mathrm{~g}$ for 10 minutes and were then washed 3 times with lysis buffer, followed by washing with buffer without Triton X-100. The inclusion bodies were dissolved in $6 \mathrm{M} \mathrm{GuHCl}, 0.5 \mathrm{mM}$ EDTA, and 10 mM DTT, 20 mMTris, $\mathrm{pH} 7.4$ and added drop-wise into a refolding buffer consisting of $1 \mathrm{M}$ Arg hydrochloride, $0.1 \mathrm{M}$ Tris, $\mathrm{pH}$ 8.0, 2mM EDTA, $0.25 \mathrm{mM}$ oxidized glutathione, $0.25 \mathrm{mM}$ reduced glutathione and $0.1 \mathrm{mg} / \mathrm{ml}$ of atezolizumab. The PD-L1 IgV domain/atezolizumab complexes were subsequently purified using ion exchange and gel filtration columns (GE Healthcare).

\section{Crystallization of PD-L1/atezolizumab complex}

PD-L1/atezolizumab complex was concentrated to $\sim 10 \mathrm{mg} / \mathrm{ml}$ and screened for crystallization conditions 
using commercially available buffers with sitting-drop vapor diffusion method where $0.2 \mu \mathrm{l}$ of the protein solution was mixed with $0.2 \mu \mathrm{l}$ of reservoir solution. Diffractionquality crystals of PD-L1/atezolizumab complex were obtained at room temperature from $2 \mathrm{M}$ ammonium sulfate and $0.1 \mathrm{M}$ Tris $\mathrm{pH} 7.0$ after optimization.

\section{Structure determination and refinement}

Crystals were cryoprotected in 20\% glycerol in the mother liquor and flash-cooled in liquid nitrogen. Diffraction data were collected on beamlines BL17U at SSRF, Shanghai, China. The data were indexed and processed with iMosflm and scaled with Aimless from the CCP4 suite [29]. The structure was solved by Phaser [30] using PD-L1 and atezolizumab models derived from PDB entries 5JDR and 5GGT, respectively. The models were subsequently manually built using Coot and refined using PHENIX [31, 32]. Figures were produced with PyMOL software [33]. The atomic coordinates and the structure factors have been deposited in the Protein Data Bank with code $5 \mathrm{XXY}$. The interface of protein structure was analyzed by PISA [34].

\section{Dissociation rate constant}

The binding kinetics of PD-L1 variants to atezolizumab was measured as previously described [19]. Briefly, all sensors were activated in PBS with $0.1 \% \mathrm{w} / \mathrm{v}$ bovine serum albumin (BSA) by agitating 96-well microtiter plates at $1,000 \mathrm{rpm}$ to minimize nonspecific interactions. Probes were soaked with 10 $\mu \mathrm{g} / \mathrm{ml}$ atezolizumab for 40 seconds before equilibration for 60 seconds in PBS with $0.1 \%$ BSA. Variants of PDL1 were prepared as a 2-fold serial dilution in PBS with $0.1 \%$ BSA and separately incubated with the atezolizumab bound on the tips for 120 seconds. Then, the PD-L1 variants were allowed to dissociate for up to 320 seconds. All measurements were corrected for baseline drift by subtracting a control sensor exposed to running buffer only. Data analysis and curve fitting were carried out using Octet software.

\section{Author contributions}

F.Z, X.Q. and X.W prepared the protein, crystallized the complex. D.W., J.W., L.F., H.C., Y.W. and Z.Z collected the data and analyzed the data. T.X, Y.Z. and A.Z designed all the experiments. All authors contributed to the manuscript preparation.

\section{ACKNOWLEDGMENTS}

We thank the staff of BL17U beamline at Shanghai Synchrotron Radiation Facility for assistance with data collection.

\section{CONFLICTS OF INTEREST}

The authors declare no conflicts of interest related to the publication of this work.

\section{FUNDING}

This work was supported in part by grants from the National Natural Science Foundation of China (31370727, 31170724, 81502981and 81572090), the National Basic Research Program of China (973 Program, 2014CB910304), the Program for Professor of Special Appointment (Eastern Scholar) at Shanghai Institutions of Higher Learning, Shanghai PuJiang Program, and Innovation Program of Shanghai Municipal Education Commission (NO.12ZZ113).

\section{REFERENCES}

1. Chen L, Flies DB. Molecular mechanisms of $\mathrm{T}$ cell co-stimulation and co-inhibition. Nat Rev Immunol. 2013; $13: 227-242$.

2. Greenwald RJ, Freeman GJ, Sharpe AH. The B7 family revisited. Ann Rev Immunol. 2005; 23:515-548.

3. Lenschow DJ, Walunas TL, Bluestone JA. CD28/B7 system of T cell costimulation. Ann Rev Immunol. 1996; 14:233-258.

4. Carreno BM, Collins M. The B7 family of ligands and its receptors: new pathways for costimulation and inhibition of immune responses. Ann Rev Immunol. 2002; 20:29-53.

5. Dong H, Zhu G, Tamada K, Chen L. B7-H1, a third member of the B7 family, co-stimulates T-cell proliferation and interleukin-10 secretion. Nat Med. 1999; 5:1365-1369.

6. Dong H, Strome SE, Salomao DR, Tamura H, Hirano F, Flies DB, Roche PC, Lu J, Zhu G, Tamada K, Lennon VA, Celis E, Chen L. Tumor-associated B7-H1 promotes T-cell apoptosis: a potential mechanism of immune evasion. Nat Med. 2002; 8:793-800.

7. Hino R, Kabashima K, Kato Y, Yagi H, Nakamura M, Honjo T, Okazaki T, Tokura Y. Tumor cell expression of programmed cell death-1 ligand 1 is a prognostic factor for malignant melanoma. Cancer. 2010; 116:1757-1766.

8. Iwai Y, Ishida M, Tanaka Y, Okazaki T, Honjo T, Minato $\mathrm{N}$. Involvement of PD-L1 on tumor cells in the escape from host immune system and tumor immunotherapy by PD-L1 blockade. Proc Natl Acad Sci U S A. 2002; 99:12293-12297.

9. Okazaki T, Honjo T. The PD-1-PD-L pathway in immunological tolerance. Trends Immunol. 2006; 27:195-201.

10. Chen L, Han X. Anti-PD-1/PD-L1 therapy of human cancer: past, present, and future. J Clin Invest. 2015; 125:3384-3391. 
11. Pardoll DM. The blockade of immune checkpoints in cancer immunotherapy. Nat Rev Cancer. 2012; 12:252-264.

12. Gunturi A, McDermott DF. Nivolumab for the treatment of cancer. Expert Opin Investig Drugs. 2015; 24:253-260.

13. Powles T, Eder JP, Fine GD, Braiteh FS, Loriot Y, Cruz C, Bellmunt J, Burris HA, Petrylak DP, Teng SL, Shen X, Boyd Z, Hegde PS, et al. MPDL3280A (anti-PD-L1) treatment leads to clinical activity in metastatic bladder cancer. Nature. 2014; 515:558-562.

14. Garon EB, Rizvi NA, Hui R, Leighl N, Balmanoukian AS, Eder JP, Patnaik A, Aggarwal C, Gubens M, Horn L, Carcereny E, Ahn MJ, Felip E, et al. Pembrolizumab for the treatment of non-small-cell lung cancer. New Engl J Med. 2015; 372:2018-2028.

15. Herbst RS, Soria JC, Kowanetz M, Fine GD, Hamid O, Gordon MS, Sosman JA, McDermott DF, Powderly JD, Gettinger SN, Kohrt HE, Horn L, Lawrence DP, et al. Predictive correlates of response to the anti-PD-L1 antibody MPDL3280A in cancer patients. Nature. 2014; 515:563-567.

16. Liu K, Tan S, Chai Y, Chen D, Song H, Zhang CW, Shi Y, Liu J, Tan W, Lyu J, Gao S, Yan J, Qi J, Gao GF. Structural basis of anti-PD-L1 monoclonal antibody avelumab for tumor therapy. Cell Res. 2017; 27:151-153.

17. Tan S, Liu K, Chai Y, Zhang CW, Gao S, Gao GF, Qi J. Distinct PD-L1 binding characteristics of therapeutic monoclonal antibody durvalumab. Protein Cell. 2017.

18. Lee JY, Lee HT, Shin W, Chae J, Choi J, Kim SH, Lim H, Won Heo T, Park KY, Lee YJ, Ryu SE, Son JY, Lee JU, Heo YS. Structural basis of checkpoint blockade by monoclonal antibodies in cancer immunotherapy. Nat Commun. 2016; $7: 13354$.

19. Zhang F, Wei H, Wang X, Bai Y, Wang P, Wu J, Jiang X, Wang Y, Cai H, Xu T, Zhou A. Structural basis of a novel PD-L1 nanobody for immune checkpoint blockade. Cell Discov. 2017; 3:17004.

20. Zak KM, Kitel R, Przetocka S, Golik P, Guzik K, Musielak B, Domling A, Dubin G, Holak TA. Structure of the complex of human programmed death 1 , PD-1, and its ligand PD-L1. Structure. 2015; 23:2341-2348.

21. Wang W, Erbe AK, Hank JA, Morris ZS, Sondel PM. NK cell-mediated antibody-dependent cellular cytotoxicity in cancer immunotherapy. Front Immunol. 2015; 6:368.

22. Tan S, Zhang H, Chai Y, Song H, Tong Z, Wang Q, Qi J, Wong G, Zhu X, Liu WJ, Gao S, Wang Z, Shi Y, et al. An unexpected N-terminal loop in PD-1 dominates binding by nivolumab. Nat Commun. 2017; 8:14369.
23. Romano E, Kusio-Kobialka M, Foukas PG, Baumgaertner P, Meyer C, Ballabeni P, Michielin O, Weide B, Romero $\mathrm{P}$, Speiser DE. Ipilimumab-dependent cell-mediated cytotoxicity of regulatory $\mathrm{T}$ cells ex vivo by nonclassical monocytes in melanoma patients. Proc Natl Acad Sci U S A. $2015 ; 112: 6140-6145$.

24. He M, Chai Y, Qi J, Zhang CW, Tong Z, Shi Y, Yan J, Tan $\mathrm{S}$, Gao GF. Remarkably similar CTLA-4 binding properties of therapeutic ipilimumab and tremelimumab antibodies. Oncotarget. 2017; 8:67129-67139. https://doi.org/10.18632/ oncotarget. 18004.

25. Clackson T, Wells JA. A hot spot of binding energy in a hormone-receptor interface. Science. 1995; 267:383-386.

26. Thanos CD, DeLano WL, Wells JA. Hot-spot mimicry of a cytokine receptor by a small molecule. Proc Natl Acad Sci U S A. 2006; 103:15422-15427.

27. Zak KM, Grudnik P, Guzik K, Zieba BJ, Musielak B, Dömling A, Dubin G, Holak TA. Structural basis for small molecule targeting of the programmed death ligand 1 (PD-L1). Oncotarget. 2016; 7:30323-30335. https://doi. org/10.18632/oncotarget.8730.

28. Guzik K, Zak KM, Grudnik P, Magiera K, Musielak B, Törner R, Skalniak L, Dömling A, Dubin G, Holak TA. Small-molecule inhibitors of the Programmed Cell Death-1/ Programmed Death-Ligand 1 (PD-1/PD-L1) interaction via transiently induced protein states and dimerization of PD-L1. J Med Chem. 2017; 60:5857-5867.

29. Collaborative Computational Project, Number 4. The CCP4 suite: programs for protein crystallography. Acta Crystallog D Biol Crystallogr. 1994; 50:760-763.

30. McCoy AJ, Grosse-Kunstleve RW, Storoni LC, Read RJ. Likelihood-enhanced fast translation functions. Acta Crystallog D Biol Crystallogr. 2005; 61:458-464.

31. Emsley P, Cowtan K. Coot: model-building tools for molecular graphics. Acta Crystallog D Biol Crystallog. 2004; 60:2126-2132.

32. Adams PD, Afonine PV, Bunkoczi G, Chen VB, Davis IW, Echols N, Headd JJ, Hung LW, Kapral GJ, GrosseKunstleve RW, McCoy AJ, Moriarty NW, Oeffner R, et al. PHENIX: a comprehensive Python-based system for macromolecular structure solution. Acta Crystallog D Biol Crystallog. 2010; 66:213-221.

33. Schrodinger, LLC. (2015). The PyMOL Molecular Graphics System, Version 1.8.

34. Krissinel E, Henrick K. Inference of macromolecular assemblies from crystalline state. J Mol Biol. 2007; 372:774-797. 